\title{
Temporal dynamics of associative interference and facilitation produced by visual context
}

\author{
G. ROBERT GRICE, JOSEPH M. BOROUGHS, and LYN CANHAM \\ University of New Mexico, Albuquerque, New Mexico
}

\begin{abstract}
Temporal dynamics of associative interference and facilitation have been investigated in a reaction time (RT) task in which a target letter is flanked by noise letters. When responseincompatible noise letters precede the target by stimulus onset asynchronies (SOAs) from 0 to $250 \mathrm{msec}$, associative interference, indicated by average RT, increases to a maximum at 50 and $100 \mathrm{msec}$ and decreases substantially at $250 \mathrm{msec}$. Noise letters identical to the target do not produce facilitation with 0 SOA but do so when they precede the target by as little as 50 msec. Maximum facilitation is obtained with 100 - and 250 - msec SOAs. Temporal dynamics within the trial were inferred from scaling analyses of the RT distributions. The time course of associative interference is nonmonotonic, with the maximum occurring earlier as SOA increases. This confirms a prediction of continuous growth strength theory. Facilitation is primarily a short-latency effect, beginning earlier as SOA increases. At $0 \mathrm{SOA}$, there is evidence that an identical noise letter produces distraction as compared with a no-noise condition.
\end{abstract}

There are two approaches to the investigation of the temporal dynamics of interference and facilitative effects in reaction time (RT) experiments in which a target is accompanied by other stimuli in a visual display. The most common of these is to investigate mean RT with manipulation of the stimulus onset asynchrony (SOA) temporally separating the target and accompanying stimuli. This method has been used by Taylor (1977), C. W. Eriksen and Schultz (1979), and Flowers and Wilcox (1982). In the other approach, use is made of information provided by the entire RT distribution in an effort to make inferences about the dynamics of processes operating within the trial. Grice, Canham, and Schafer (1982a) applied the scaling methods of variable criterion theory to the cumulative distribution and obtained functions describing the growth of associative strength following stimulus onset. Their work was confined to 0 SOA. It should be understood that the two approaches investigate different aspects of temporal dynamics and should not be regarded as alternative ways of obtaining the same information. The present research uses the procedures in combination to obtain both kinds of information simultaneously. In other words, the question is raised as to how variations of SOA affect the temporal dynamics of information processing during a trial.

Specifically, the present research is concerned with choice reaction-time (CRT), letter-identification experiments in which a central target letter is flanked, horizontally and symmetrically, by noise letters. Fixation is directed to the central target, and subjects are instructed

Portions of this research were supported by PHS Grant MH 16400 from the National Institute of Mental Health. Requests for reprints should be sent to G. Robert Grice, Department of Psychology, University of New Mexico, Albuquerque, NM 87131. to ignore the flanking letters. B, A. Eriksen and C. W. Eriksen (1974) discovered that if the noise letters in this situation were response-incompatible with the target, RT was slowed relative to conditions with responsecompatible or irrelevant noise letters. Their work was with simultaneous onset of target and noise. C. W. Eriksen and Shultz (1979) found that the compatibility effect increased to a maximum when noise letters preceded the target by 100 -msec SOA and then decreased to very little at $250 \mathrm{msec}$. Their research was not designed to determine interference and facilitative effects separately, but their general pattern of results suggests that interference was predominant. In a more complicated experiment designed to investigate additional variables, Flowers and Wilcox (1982) obtained quite similar results, but did produce evidence of facilitation by noise letters identical to the target when noise preceded the target. Our research is limited to identity for response-compatible noise, and other manipulations of the response-compatible condition are not considered here.

In five experiments with simultaneous onset of target and noise letters, Grice et al. (1982a) examined three noise conditions: noise identical to the target, noise as an irrelevant letter, and noise response-incompatible with the target. In each experiment, they found the irrelevantnoise-condition RTs to be significantly slower than those for identical noise, and incompatible-noise RTs to be significantly slower than those for irrelevant noise. The difference between identical and irrelevant noise was termed perceptual interference and is attributed to a distraction effect produced by the irrelevant noise letters. Although a similar difference was attributed by Taylor (1977) to facilitation by identical noise, the interference interpretation is preferred because there is consistent evidence that, when compared with an appropriate no-noise 
control condition, identical noise does not produce facilitation (B. A. Eriksen \& C. W. Eriksen, 1974; C. W. Eriksen \& B. A. Eriksen, 1979; C. W. Eriksen \& Schultz, 1979). The difference between irrelevant and incompatible noise was attributed to associative interference. Irrelevant noise is the appropriate control condition for measuring the associative interference produced by response-incompatible noise. Both conditions involve noise letters different from the target, and thus produce the distraction effect. The additional slowing for incompatible noise is the specific interference produced by response-incompatibility. In experiments manipulating the SOA, the only research including this comparison is that of Taylor (1977). However, his procedures differed in other ways from the research considered here.

Grice et al. (1982a) also used the scaling procedures of variable criterion theory to investigate the temporal dynamics of these processes during a trial. According to this view, the excitatory or associative strength leading to response evocation grows as a continuous, orderly function of the time following stimulus onset. When this growing strength reaches a criterion level, the response is evoked. The time of evocation varies from trial to trial, because the criterion is assumed to be a normally distributed random variable. This model makes it possible to obtain accurate estimates of the form of the growth functions by applying Thurstonean scaling procedures to the data of cumulative RT distributions. ${ }^{1}$

When the measurement model was applied to the data, it was determined that the growth of excitatory strength (E) for the identical noise condition was a simple negatively accelerated function that was described by an exponential growth function of the form.

$$
E(t)=a-m e^{-k t},
$$

where $\mathrm{a}, \mathrm{m}$, and $\mathrm{k}$ are empirical constants and $\mathrm{t}$ is the time, in milliseconds, following stimulus onset. This function was interpreted as describing the growth of associative strength (A) leading to response evocation. In terms of the theory, the more complete statement is

$$
\mathrm{E}(\mathrm{t})=[\mathrm{A}(\mathrm{t})-\overline{\mathrm{C}}] / \sigma,
$$

where $A(t)$ is the value of Equation 1 at time $t$, and $\bar{C}$ and $\sigma$ are the mean and standard deviation of the normal, criterion distribution. When the function has been estimated directly from a set of data, the values of $\bar{C}$ and $\sigma$ are 0 and 1 . The growth function for irrelevant noise was identical in form to that for identical noise except for a small subtractive constant. Thus, the distraction or perceptual interference effect did not affect the shape of the growth function, but reduced it by a constant throughout. It may be noted from Equation 2 that the model is not capable of distinguishing between a criterion effect and a purely perceptual one if the effect is simply additive. Generally, when stimuli are presented in an unpredictable order, as these conditions were, all should be based on the same criterion distribution. Of course, it could be argued that the very short latency effect of nonidentical noise is a rapid elevation of the criterion. Although this is possible, it was not suggested by the authors.

The growth function for incompatible noise was of different form. The time-course of associative interference (AI) was determined from the difference between this function and that for irrelevant noise. The resulting function for AI was nonmonotonic. Associative interference was near zero at the shortest latencies, increased to a maximum between 400 and $500 \mathrm{msec}$, and then gradually decreased to near zero at long latencies above $700 \mathrm{msec}$. The function was described by an equation based on the derivative of the Gompertz growth function. A hypothesis to account for this function concerned error tendencies produced by incompatible noise letters. As such tendencies near criterion, they may be inhibited. However, the inhibition process also delays correct responses approaching criterion at about the same time. The declining phase of inhibition was interpreted as a recovery period, during which responses delayed earlier then occur. During this phase, growth is more rapid for incompatible noise than for the control condition, irrelevant noise. Grice, Canham, and Schafer (1982b) reported one kind of evidence tending to support this interpretation. In disjunctive reaction time (DRT) experiments, subjects were instructed to respond to one target but not the other. In this situation, associative interference was not obtained, suggesting the necessity of an active, competing response. Although this evidence is not regarded as conclusive, it is true that if the competing response is not responding, there is nothing to inhibit.

The nonmonotonic time course of associative interference naturally raises the question as to whether there are experimental variables that might influence the time of maximum interference. The SOA is an obvious possibility for which continuous growth theory makes a specific prediction. If the noise stimuli precede the target by an SOA greater than zero, then priming of the competing response should begin earlier, and the growth of error tendencies should occur sooner. According to the above reasoning, this implies that maximum interference should occur earlier, when noise precedes the target. Within limits, at least, the maximum should occur progressively earlier as SOA is increased. It should be noted that this is not a prediction shared by theories conceiving of information processing as a sequence of discrete stages. The relation between the portion of an RT distribution primarily affected and a change in any particular stage is a complex matter (McGill \& Gibbon, 1965), and there are not stage theories presently in existence capable of making a prediction about the relationship. This research tests the prediction of continuous growth theory, but not a differential prediction with respect to stage theory. In view of the basic similarity of the continuous flow conception of C. W. Eriksen and Schultz (1979), we believe that the prediction follows from that view as well.

The research here consists of two experiments, each consisting of four subexperiments. The subexperiments in each experiment consist of separate groups of subjects, 
and employ a different SOA by which noise letters precede the target. These values are $0,50,100$, and $250 \mathrm{msec}$. Each subexperiment includes four noise conditions: noise letter identical to the target, noise letter irrelevant, noise letter response-incompatible with the target, and a condition with no noise letter. The two full experiments differ only in the nature of the condition with no noise letter.

One variable affecting the compatibility effect in this situation is the horizontal spacing between target and noise (B. A. Eriksen \& C. W. Eriksen, 1974). The spacing used here is $.5^{\circ}$. This spacing was used by $\mathrm{C}$. W. Eriksen and Schultz (1979) because it is sufficient to avoid contour interference and still produces a substantial compatibility effect. It was also used by Grice et al. (1982a).

\section{EXPERIMENT 1}

If interference or facilitation effects of identical noise letters are to be evaluated, an appropriate control condition is necessary. With simultaneous onset of target and noise it seems clear that the best comparison is with a simple no-noise condition. The status of this condition with nonzero SOAs is less clear. Since only the target letter is presented, no SOA is involved. In nonzero SOA conditions, this means a mixture of trials with and without noise preceding the target. This suggests the possibility of differential preparation on trials with and without noise. The question is an empirical one and of some interest in its own right. The decision was to include the simple nonoise condition in all SOA conditions in the initial experiment.

\section{Method}

Subjects. The subjects were 112 undergraduate women from courses in introductory psychology. They received course credit for participation. There were 28 subjects in each of the four subexperiments.

Apparatus and Procedure. The experimental situation was as described by Grice et al. (1982a). The experiment was controlled and data collected by a TRS- 80 Model I microcomputer equipped with a millisecond clock and interface circuitry. Letter stimuli were the regular double-width letters produced by the computer and were formed in a $5 \times 7$ dot matrix on a video monitor. At the viewing distance used, the letters were approximately $.53^{\circ}$ high and $.43^{\circ}$ wide. A plus sign appeared in the center of the screen at all times except when letters were present. The target letter appeared $.45^{\circ}$ directly above the fixation point. Stimulus presentation and timing were synchronized with the vertical scan of the screen, and SOAs were timed by counting the pulse controlling the vertical scan (Grice, 1981). Responses were made by pressing, with the left or right index finger, one of two telegraph keys on a table before the subject. RTs were recorded in milliseconds.

The target letters were $A$ and $H$. Half of the subjects responded to A with the left key and $\mathbf{H}$ with the right key. The other half of the subjects had the reverse arrangement. On noise trials, the same letter appeared on both sides of the target at a distance of $.5^{\circ}$. In each of the four SOA conditions, there were four noise conditions: Target alone, noise same as target, noise an irrelevant letter (K), and noise the letter mapped on the other response (response incompatible). The SOA conditions were administered to separate groups of subjects. These conditions were target and noise simultaneous
( 0 SOA) and noise preceding the target by 50,100 , and $250 \mathrm{msec}$ On noise trials, the fixation point went off when the noise letters appeared. On no-noise trials, it went off when the target appeared. All letters went off and the fixation point reappeared when the subject responded.

One second before each trial there was an auditory warning signal, a .5-sec $1000-\mathrm{Hz}$ tone of $70 \mathrm{db}$, presented by earphones. The subjects were instructed then to look at the fixation point and get ready to respond. They were instructed to respond to the target as quickly as possible while avoiding errors. They were also instructed to ignore the letters flanking the target. On trials with errors, the word ERROR appeared on the screen and a $70-\mathrm{dB}$ noise sounded in the earphones for $.5 \mathrm{sec}$. The interval between trials was $4 \mathrm{sec}$. Each kind of noise occurred equally often, as did each target with each noise type. The order of conditions and targets was irregular.

The experiment began with 50 practice trials followed by a short rest. At this time, any questions were answered and any necessary reinstruction given. This was followed by 250 trials without interruption. The first 10 of these were treated as warm-up and as further practice. Data were based on the final 240 trials, 60 with each noise condition.

\section{Results and Discussion}

Means of the subject median RTs for correct responses, for each noise condition in each of the four SOA groups, are presented in the upper portion of Figure 1. Means presented essentially the same pattern, but between-subject variability was somewhat less for the medians. Error rates were low. For the four noise conditions, none, identical, irrelevant, and incompatible, the mean rates were .008 , $.009, .013$, and .038 , respectively.

At 0 SOA, the identical, irrelevant, and incompatible noise conditions replicated the findings of Grice et al. (1982a). The associative interference effect based on the difference between incompatible and irrelevant noise was

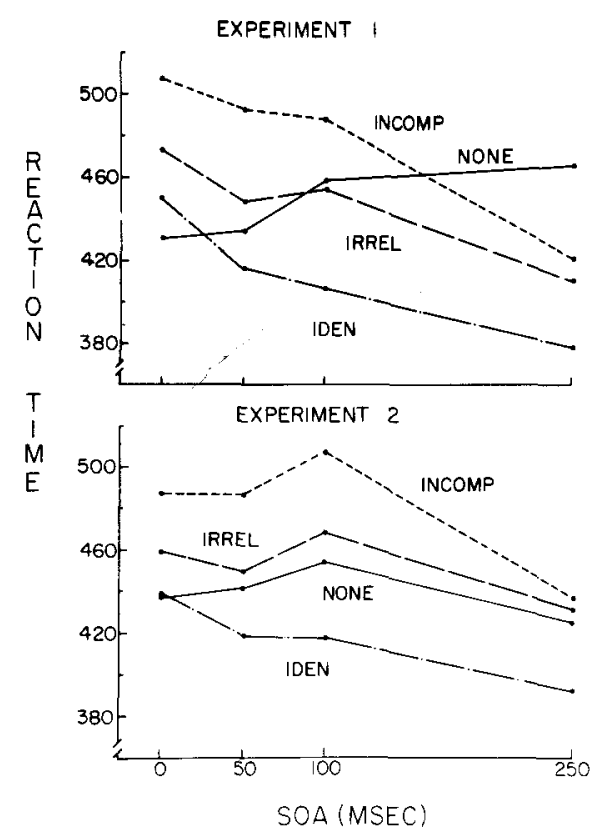

Figure 1. Means of subjects' median reaction times in milliseconds in Experiments 1 and 2 for each noise letter condition at each SOA. 
significant $[\mathrm{t}(27)=7.648, \mathrm{p}<.001]$. The difference between identical and irrelevant noise, referred to as perceptual interference in the previous article, was also significant $[\mathrm{t}(27)=6.190, \mathrm{p}<.001]$. The no-noise condition was included here to provide a control for evaluating facilitation or interference effects of identical noise. The results show that the effect was clearly interference. The no-noise condition was $19 \mathrm{msec}$ faster than identicalnoise condition, and the difference is significant $[\mathrm{t}(27)=$ $6.311, \mathrm{p}<.001$ ]. This result is quite consistent over subjects. Only 4 of the 28 subjects were faster with identical than with no noise, and these only by an average of $5 \mathrm{msec}$. The finding of a clear interference or distraction effect of identical noise is a little surprising, since the typical finding has been that RTs with no noise and identical noise were about the same (B. A. Eriksen \& C. W. Eriksen, 1974, 1979; C. W. Eriksen \& Schultz 1979). However, this experiment is not a replication of any of the previous ones. Also, each data point here is based on a substantially larger number of observations than in any of the previous research. In spite of evidence for an inhibitory effect of identical flankers, the data do not support the feature-specific inhibition hypothesis of Bjork and Murray (1977). That view would predict irrelevant noise RT to be faster than identical noise RT. Consistent with previous findings at 0 SOA, the obtained difference was significant in the opposite direction.

Associative interference, inferred from the difference between incompatible and irrelevant noise, is present at all SOAs. For this comparison, the primary effect of noise type is significant $[\mathrm{F}(1,108)=220.26, \mathrm{p}<.001]$. However, the interaction of noise type with SOA is also significant $[F(3,108)=12.43, p<.001]$. This reflects, primarily, the substantially reduced size of the effect at $250-\mathrm{msec}$ SOA. The finding is in accord with that of C. W. Eriksen and Schultz (1979), as well as that of Flowers and Wilcox (1982).

The most striking feature of the total pattern of the data is the increase of RT for the no-noise condition with increasing SOAs, while RTs for the other condition show decreasing trends. The best interpretation of this pattern is in terms of a criterion of preparedness effect. At nonzero SOAs, noise letters preceded the target on threefourths of the trials. If the noise letters play some role as a warning or alerting signal, then there should be less preparedness on those trials when the target appears alone at the time of the noise letters. This leads to the prediction that preparedness for the target with no noise should be best in the 0 SOA group and worst in the 250 SOA group. A comparison of these two conditions supports the prediction $[\mathrm{t}(54)=1.756, \mathrm{p}<.05$, one-tailed test $]$. In a comparison of the no-noise and irrelevant-noise conditions, the interaction of noise type with SOA is significant $[F(3,108)=74.04, p<.001]$. Differential preparedness as a function of SOA appears to be the most likely basis for this strong interaction.

Another interesting feature of the data is the comparison of the identical and no-noise conditions at 0 and 50 SOA. Although identical noise is slower at 0 SOA, the relation is reversed at $50 \mathrm{msec}$. This interaction is significant $[F(1,54)=50.21, p<.001]$, and is a surprisingly strong effect for only a 50-msec manipulation. Unfortunately, it is impossible to determine how much of the reversal is due to criterion elevation for no noise or facilitation by the identical flankers. The consistent decrease with SOA of RT for identical noise suggests a facilitation effect. However, it is clear that the present no-noise condition provides an adequate comparison only with simultaneous onset of target and flankers.

\section{EXPERIMENT 2}

To provide an adequate control condition for the evaluation of facilitation, it is necessary to present some stimulus with the same SOA as the noise letters. This neutral stimulus should be unobtrusive and not resemble a letter. What we have done in Experiment 2 for the condition without noise letters is to enclose the target in parentheses, appearing in the same location as the noise letters and with the same SOA with respect to the target. Otherwise, Experiment 2 is a replication of Experiment 1.

\section{Method}

Subjects. The subjects were 96 undergraduate women from the same population as above. There were 24 in each of the four subexperiments.

Procedure. Except for the condition without noise letters, procedures were identical to those of Experiment 1 . In this condition, a left parenthesis appeared in the location occupied by the left noise letter and a right parenthesis in the position of the right noise letter. In each of the four groups, onset of the parentheses preceded the target by the same SOA as the noise letters.

\section{Results}

As in Experiment 1, error rates were low. For the four noise conditions, none, identical, irrelevant and incompatible, the mean rates were $.008, .007, .013$, and .028 . The means of subject median RTs for correct responses are presented in the lower portion of Figure 1.

The most obvious difference in the pattern of the two experiments is that the no-letter-noise condition with parentheses does not increase with SOA. Rather, it remains consistently below the irrelevant noise condition. At 0 SOA, the no-noise condition is about equal to identical noise rather than faster. This differs significantly from Experiment 1 . In comparing the two experiments, the noise type $\times$ experiments interaction is significant $[F(1,50)=18.08, p<.001]$. This suggests that the parentheses produce a small distraction effect, approximately equal to that of an identical noise letter. Also with 0 SOA, the difference in the two no-noise conditions did not affect the relation between the remaining three noise conditions. In comparing identical, irrelevant, and incompatible noise across experiments, the primary effect of experiments was not significant $[F(1,5)<1]$. Also, the interaction of experiments and noise conditions was not significant $[F(2,100)<1]$. The effect of noise condition was significant $[\mathrm{F}(2,100)=130.76, \mathrm{p}<.001]$.

The distraction effect of an irrelevant noise letter is in- 
terpreted as the difference between the irrelevant and noletter-noise conditions. Recognizing that the parentheses may have a small distraction effect, this should be interpreted as the additional effect of an irrelevant letter. The effect is plotted as a function of SOA in Figure 2. Across SOAs, the primary effect is significant $[F(1,92)=30.89$, $\mathrm{p}<.001]$. The difference is greatest with simultaneous onset and least at $250 \mathrm{SOA}$. However, the interaction with SOA is of only borderline significance $[\mathrm{F}(3,92)=2.58$, $.05<\mathrm{p}<.10]$. There is some evidence that the difference between 0 and 250 SOA is real since 20 of 24 subjects were faster to no noise at 0 SOA but only 12 of 24 at $250 \mathrm{SOA}$. This difference in proportion is significant $\left[\chi^{2}(1)=4.95, p<.05\right]$. In comparing this relation between experiments, the three-way interaction of noise $X$ SOA $\times$ experiments is significant $[F(3,200)=40.10$, $\mathrm{p}<.001]$. This supports the hypothesis that, in Experiment 1 , the no noise condition led to decreasing preparation with increasing SOAs.

Associative interference is indicated by the difference between irrelevant and incompatible noise. This effect is also plotted as a function of SOA in Figure 2. The effect is significant $[\mathrm{F}(1,92)=134.81, \mathrm{p}<.001]$. Also, it increases to a maximum at 50 and $100 \mathrm{SOA}$ and then decreases to a small amount at 250 SOA. The interaction of noise $\times$ SOA is significant $[F(3,92)=10.75$, $\mathrm{p}<.001]$. These results replicate those of Experiment 1, and generally agree with previous findings, although this particular comparison was not made by $\mathrm{C}$. W. Eriksen and Schultz (1979) or by Flowers and Wilcox (1982). In an analysis of these two conditions in Experiments 1 and 2 , none of the comparisons involving experiments were significant. The results of these tests were as follows: experiments, $F(1,200)<1$; experiments $\times$ SOA, $F(3,200)$ $=1.00$, n.s.; noise $\times$ experiments, $F(1,200)=1.23$,

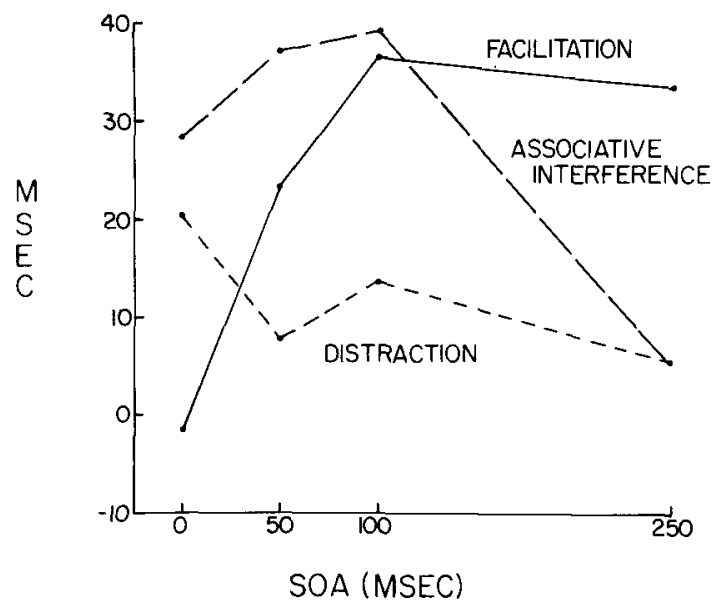

Figure 2. Facilitation, associative interference, and distraction effects in milliseconds for Experiment 2, based on the data of Figure 1. Facilitation = no-noise-letter RT - identical noise letter RT. Associative interference = incompatible noise letter RT - irrelevant noise letter RT. Distraction = irrelevant noise letter RT - no noise letter RT. n.s.; noise $\times$ SOA $\times$ experiments, $F(3,200)<1$. In the combined analysis for the effect of noise, $F(1,200)=$ $336.52, \mathrm{p}<.001$; and for noise $\times \operatorname{SOA}, \mathrm{F}(3,200)=$ $21.69, \mathrm{p}<.001$.

In Experiment 1, because of the problem of decreasing preparation, the no-noise condition provided an appropriate comparison with identical noise only at 0 SOA. The use of the parentheses in Experiment 2 eliminated this problem, and the no-letter-noise condition provides a basis for examining interference or facilitation effects. The difference between no noise and identical noise as a function of SOA is included in Figure 2. At 0 SOA, the difference is only $2 \mathrm{msec}$ and neither effect is indicated. Beyond that, the effect is clearly facilitative. Facilitation increases rapidly to a maximum at $100 \mathrm{msec}$ and is about the same at $250 \mathrm{msec}$. The primary effect of noise condition is significant $[\mathrm{F}(1,92)=119.16, \mathrm{p}<.001]$, as is the interaction of noise and $\operatorname{SOA}[\mathrm{F}(3,92)=17.33$, $\mathrm{p}<.001]$. An SOA of only $50 \mathrm{msec}$ produces facilitation of about 24 msec compared with none of 0 SOA. In a comparison of these two SOAs, the noise $\times$ SOA interaction is significant $[\mathrm{F}(1,46)=26.20, \mathrm{p}<.001]$.

\section{TEMPORAL DYNAMICS WITHIN SOAS}

Functions for the growth of excitatory strength were determined by the scaling procedures of variable criterion theory as in the research of Grice et al. (1982a). The data analyzed were the average, group distributions with cumulative proportions averaged at 20 -msec intervals. The first step was to correct all distributions for errors by application of the race model of Grice, Spiker, and Nullmeyer (1979). This yields cumulative distribution functions that are invariant with respect to error rate. However, since error rates were low, they differ little in form from the uncorrected distributions.

\section{Associative Interference}

Analysis of the effects of associative interference was based on the data of Experiments 1 and 2 jointly. The statistical analysis indicated that this effect was comparable in the two experiments, and it was desired that these functions be determined as precisely as possible. The first step was to transform the distributions for irrelevant and incompatible noise to scale values of excitatory strength. The four growth functions of Experiment 1 for irrelevant noise were fitted with exponential growth functions of the form of Equation 1. The fits were excellent. The functions for both noise conditions were then converted to the common scale of Experiment 2. This was done by linear response evocation characteristics (RECs) relating irrelevant noise for Experiment 2 to the fitted function, and the two obtained functions for incompatible noise to each other. That these relations were linear indicated that the functions were of the same form in the two experiments, and differed only by the parameters of the criterion distribution estimated by the linear relations. ${ }^{2}$. 
The time course of associative interference is given by the differences between the functions for the growth of excitatory strength for irrelevant and incompatible noise. The function for incompatible noise was obtained by averaging the obtained functions for Experiments 1 and 2 on the scale of Experiment 2. These values were then subtracted from the calculated function for irrelevant noise, on the same scale. Estimates were obtained at $20-\mathrm{msec}$ intervals. The results were four orderly nonmonotonic functions with differing maxima for the four SOAs. All of them could be accurately described by the same kind of function used by Grice et al. (1982a). This was an equation based on the derivative of the Gompertz growth function. It was written for finite $20-\mathrm{msec}$ intervals rather than as a differential equation. These fitted functions, which accurately describe the data, are presented in Figure 3. For 0 SOA, the maximum is at $453 \mathrm{msec}$, and the function essentially duplicates that obtained in the previous research. The maxima of the functions for the other SOAs are as follows: $50 \mathrm{SOA}, 373 \mathrm{msec} ; 100 \mathrm{SOA}, 361 \mathrm{msec}$; and 250 SOA, $301 \mathrm{msec}$. This confirms the prediction of continuous growth theory that maximum interference should occur progressively earlier with increases in SOA. There is also a tendency for interference to decrease more rapidly from its maximum with increasing SOAs. This is especially true at 250 SOA. C. W. Eriksen and Schultz (1979) suggested that there would be a tendency for priming of the competing response to dissipate after the onset of the noise letters.

The derivative of the Gompertz function approaches zero in the limit. This is reflected in the functions for 0 and 50 SOA in Figure 3. The limits are not exactly zero for 100 and 250 SOA. The most probable basis for this

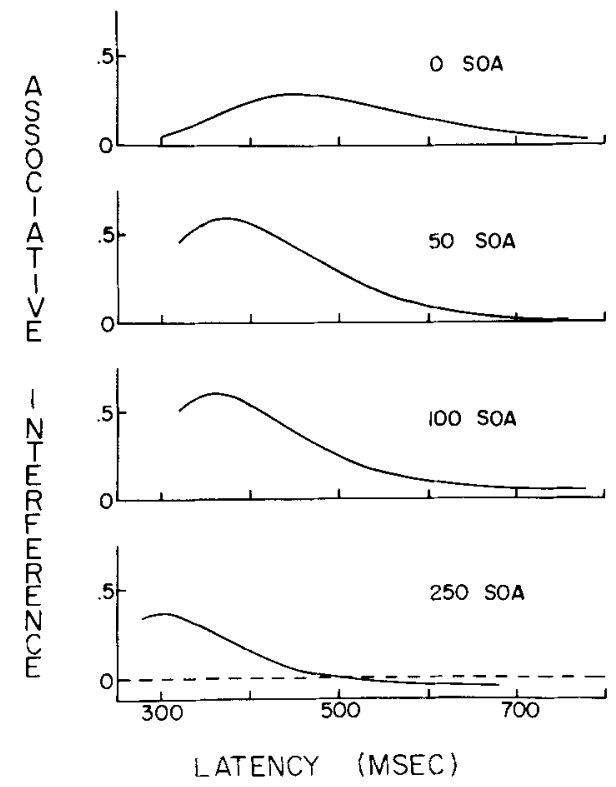

Figure 3. Calculated functions describing the time course of associative interference for each SOA. Scale units are those of Experiment 2. is small within-subjects sampling error between the criterion levels of irrelevant and incompatible noise. In order to fit the function, a small constant was added or subtracted and then removed after fitting. These constants are not present in the figures, and these functions accurately describe the data. The calculated functions for the growth of excitatory strength for incompatible noise are the differences between the exponential growth functions for irrelevant noise and the functions for associative interference in Figure 3.

\section{Distraction and Facilitation Effects}

At all SOAs, growth functions for the no-letter-noise condition and irrelevant noise were of the same form. RECs relating the two conditions were linear and consistently indicated that they differed by an additive constant with the no noise function greater than that for irrelevant noise. This constant represents the distraction effect. Differences in the slope parameters of these RECs were small and not consistent. Calculated growth functions for the no-letter-noise (parentheses) conditions consist of those for irrelevant noise conditions with the linear transformations indicated by the RECs.

Facilitation was not obtained at 0 SOA. In this case, the growth functions for the identical and no-noise conditions were approximately the same. At the other SOAs, facilitation was reflected in the shape of the growth function. While these functions were still of the form of exponential growth functions, they displayed more early development of associative strength and approached their limits more rapidly. RECs relating these functions to no noise were not linear. These three functions for identical noise were precisely fitted with exponential functions that described their more rapid growth.

Smoothed, but accurate, representations of the time course of facilitation are functions consisting of the difference between the calculated growth functions for no noise and identical noise. These functions are presented in Figure 4. At these three SOAs, facilitation is predominately a short latency effect. At 50 SOA, the effect is approximately constant for about $80 \mathrm{msec}$ and then decreases monotonically. In both the 100 - and $250-\mathrm{msec}$ conditions, facilitation is greatest at the shortest latencies and decreases monotonically throughout the distribution.

In order to measure the effect of associative interference as accurately as possible, the growth functions for irrelevant noise were fitted separately for each SOA. Actually, this was unnecessary, since all of these functions are nearly of the same form except for the criterion parameters. This aspect of the data makes it possible to obtain estimates of criterion level that are comparable across SOAs and experiments. RECs have been examined relating all of the irrelevant and no-letter-noise functions to a single calculated function. The one used was that for the 0 SOA, no-noise condition of Experiment 2. All of the values of $r^{2}$ in these linear regressions were greater than .998 . The resulting estimates of $\mathrm{C}$, the mean of the criterion distribution, are presented in Figure 5. The 


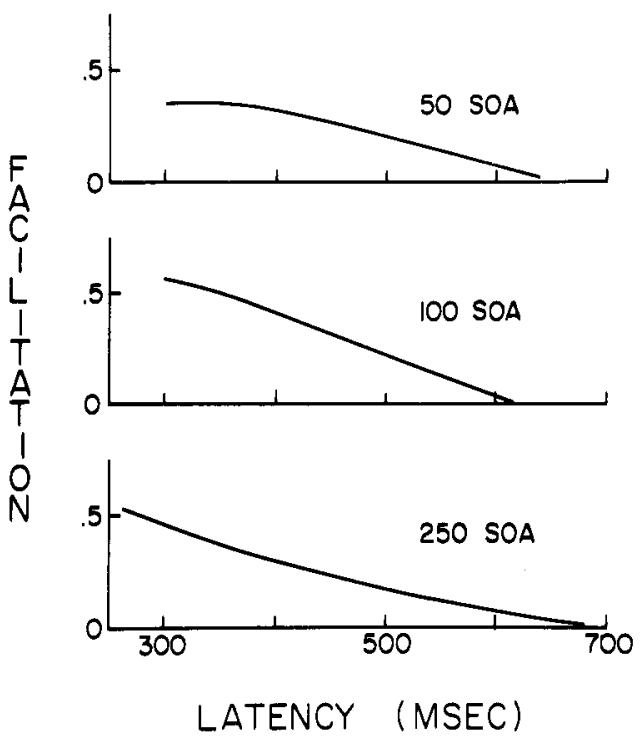

Figure 4. Calculated functions describing the time course of associative facilitation in Experiment 2 for those SOAs with which the effect was obtained.

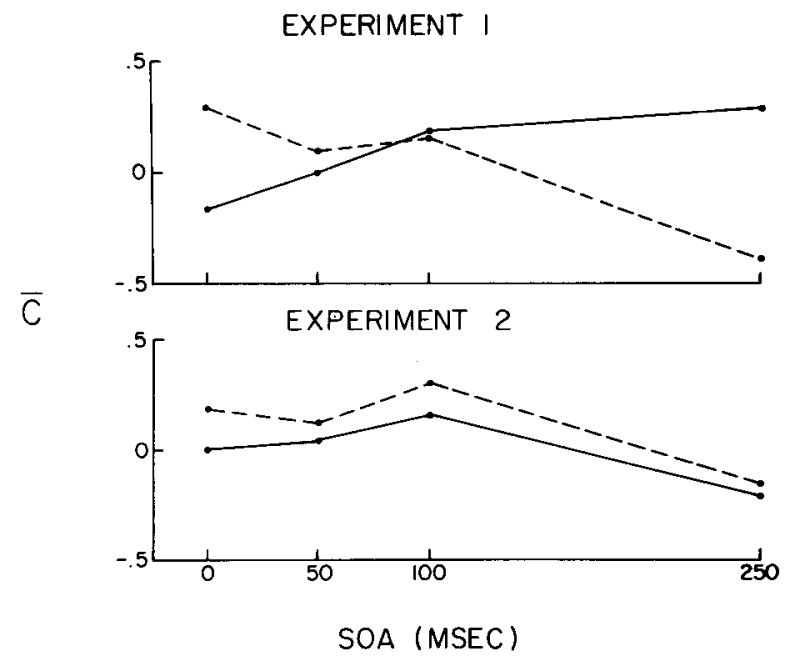

Figure 5. Mean (C) of the criterion distributions for irrelevant and no letter noise on a common scale for Experiments 1 and 2. The differences between the two functions for Experiment 2 indicate the distraction effect of irrelevant noise.

crossing functions for Experiment 1 illustrate the effect of differential preparation, that is, criterion level, that occurred with increasing SOAs. As mentioned earlier, this analysis does not differentiate between the criterion mean and other possible simple additive effects. The differences between no noise and irrelevant noise for Experiment 2 indicate the distraction effect of irrelevant noise, measured on the common scale.

\section{Goodness of Fit}

The credibility of the model presented here must depend upon the degree of accuracy with which it describes the data. In order to evaluate this, we have calculated all of the distribution functions to evaluate the fit.

The calculated values of the functions for the growth of excitatory strength for Experiment 2 are presented in Figure 6. For 0 SOA, the function for identical noise is not included since it essentially is superimposed with that for no noise. For $250 \mathrm{SOA}$, the function for irrelevant noise is omitted because it is too close to that for no noise for separate representation. Features of the functions previously mentioned are observable here. For nonzero SOAs, the early superiority of identical noise to no noise is evident. Also, the different shapes of the incompatible noise functions, dependent on associative interference, may be seen.

The normal functions along the strength axis represent the theoretical distribution of the criterion and illustrate the relation between response probability and the growth of excitatory strength. The cumulative proportion of response at any time is indicated by the area of the normal curve below the ordinate of a growth function at that time. In other words, the predicted proportion of response is the cumulative normal distribution function of $E$ at any time. In this way, fits to each of the 16 distributions have been obtained. Calculations were at 20 -msec intervals for proportions from .02 to .98 . Goodness of fit was evaluated for each distribution by the proportion of variance of the data points accounted for by the calculated values. All of these proportions were .999 or greater. The mean proportion of variance for the 16 distributions was .9994 . The model provides an extremely precise description of the data.

Fits to the distributions of Experiment 1 have also been obtained by transforming the functions of Figure 6 to the scales of the corresponding conditions of that experiment. In this analysis the proportions of variances accounted for was greater than .999 for 13 of the 16 distributions, for 2 it was .998, and for 1 it was .997 . The mean proportion was .9991 . This means that differences between the experiments may be interpreted as differences in criterion parameters and not in the form of the growth functions. It also means that these functions are replicable.

\section{DISCUSSION}

These experiments contribute information concerning two aspects of the temporal dynamics of interference and facilitation-the effect of SOA on average RT, and withintrial processes as they are affected by SOA. Concerning the first aspect, the results are based on a substantially larger number of observations per data point than previous research. Thus, the functions of Figure 1 are somewhat more reliable than any previously reported. Our work most closely resembles that of C. W. Eriksen and Schultz (1979), since we used the same spacing between letters and three of the same SOAs. Our function for incompatible noise in Experiment 2 closely resembles theirs, with RT increasing slightly to a maximum at 100msec SOA and then decreasing substantially at $250-\mathrm{msec}$. 

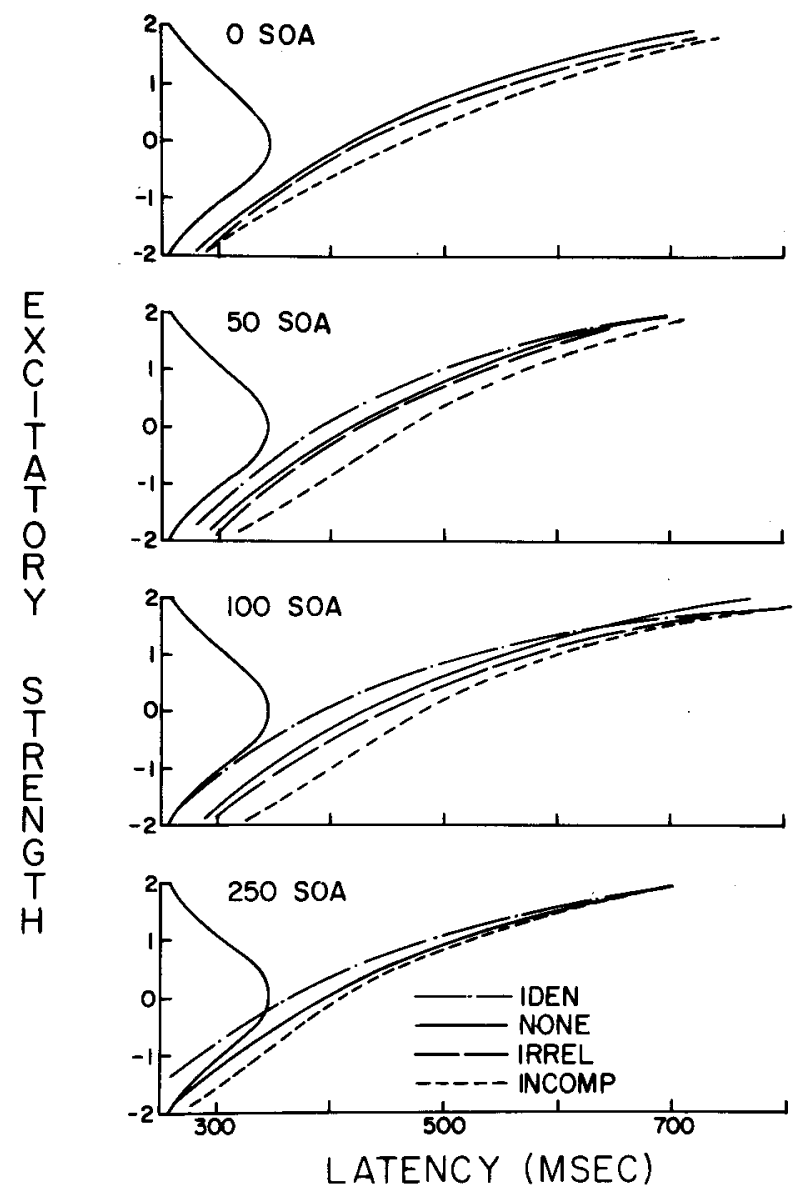

Figure 6. Calculated functions in scale units describing the growth of excitatory strength for Experiment 2. At zero SOA, the functions for identical and no letter noise were superimposed. This was also approximately true of no noise and irrelevant noise at 250 SOA. Normal distributions on the strength axis represent the criterion distribution.

However, our research isolated the function for associative interference by providing the comparison with an irrelevant noise condition. C. W. Eriksen and Schultz did not study facilitation effects. However, Flowers and Wilcox (1982) provided comparisons of three responsecompatible conditions with a condition with asterisks rather than noise letters. In agreement with our results, they found evidence of facilitation by identical noise at SOAs greater than zero, but not with simultaneous onset. However, various procedural differences make further comparison inappropriate. The procedures used by Taylor (1977) were not sufficiently similar for any meaningful comparison. The distraction effect of irrelevant noise letters has not been investigated in the previous research. Our results suggest that this form of interference decreases somewhat as SOAs increase from zero.

Temporal dynamics inferred from RT distributions in this situation are investigated for the first time in the present research. The result is a set of orderly and interpretable functions that accurately describe the data. The form of functions for the growth of associative strength is un- affected by SOA in conditions with no noise letter or an irrelevant noise letter. The form is affected at all SOAs by associative interference, but differentially so at different SOAs. The time course of interference is described by the same kind of function at all SOAs, but the maximum is progressively earlier as incompatible noise letters precede the target by longer times. This confirms the prediction of continuous growth theory. In terms of average RT, interference is minimal at 250 SOA. This reflects two aspects of dynamics within the trial: the maximum of the interference function is less than at shorter SOAs, and interference declines more rapidly. Possibly, at longer intervals, interfering responses may be inhibited more easily and at less cost.

Facilitation is produced only when the identical noise letter precedes the target. Fifty milliseconds are sufficient to produce significant facilitation, but the effect is increased with $100 \mathrm{msec}$. These results seem to imply that the priming effect of the identical letter must be already present during early processing of the target. This conclusion is reinforced by the scaling analysis, which indicates that facilitation is primarily a short-latency effect. The fact that facilitation begins earlier as SOA is increased is in accord with the natural expectation of continuous growth theory: the earlier the extra source of associative strength is available, the sooner the effect can begin. Facilitation definitely affects the form of the function for the growth of associative strength. It is not merely a displacement of the RT distribution.

A question that naturally arises concerns the effects with 0 SOA. If incompatible noise produces interference, why should identical noise not produce facilitation? Any answer to this question must recognize that in one case it is associative strength to the target that is involved, while in the other it is that of a competing response. The present data suggest that priming of response to the target depends on the prior appearance of the priming stimulus. On the other hand, they further suggest that two stimuli may activate two different responses simultaneously. Additional differences in temporal dynamics presumably depend on the interaction of two response systems. The active inhibition of the competing response may be a part of this interaction. One speculation to account for the lack of facilitation with simultaneous presentation has been mentioned by Krueger and Shapiro (1980) and, more recently, by Yeh and C. W. Eriksen (1984). This is the possibility that identical noise produces both facilitation and interference, but that the effects tend to cancel. Our analysis provides, at least circumstantial, evidence contrary to this interpretation. If associative facilitation were involved, we should expect the form of the growth function to be affected and to be different from that obtained with irrelevant or no letter noise. This was not observed. Recently, we have obtained evidence against associative facilitation by a second target letter in divided attention tasks (Grice, Canham, \& Boroughs, 1984). The evidence indicated that the "redundant targets effect" in RT experiments is due to the fact that a second target replaces an irrelevant letter that is more distracting. 


\section{REFERENCES}

BJoRK, E. L., \& MurRAY, J. T. (1977). On the nature of input channels in visual processing. Psychological Review, 84, 472-484.

EriKsen, B. A., \& EriKsen, C. W. (1974). Effects of noise letters upon the identification of a target letter in a nonsearch task. Perception \& Psychophysics, 16, 143-149.

ERIKSEN, C. W., \& ERIKSEN, B. A. (1979). Target redundancy in visual search: Do repetitions of the target within the display impair processing? Perception \& Psychophysics, 26, 195-205.

ERIKSEN, C. W., \& SCHULTZ, D. W. (1979). Information processing in visual search: A continuous flow conception and experimental results. Perception \& Psychophysics, 25, 249-263.

Flowers, J. H., \& Wilcox, N. (1982). The effect of flanking context on visual classification: The joint contribution of interactions at different processing levels. Perception \& Psychophysics, 32, 581-591.

GrICE, G. R. (1972a). Application of a variable criterion model to auditory reaction time as a function of the type of catch trial. Perception \& Psychophysics, 12, 103-107.

GRICE, G. R. (1972b). Conditioning and a decision theory of response evocation. In G. H. Bower (Ed.), The psychology of learning and motivation (Vol. 5, pp. 1-65). New York: Academic Press.

GRICE, G. R. (1981). Accurate reaction time research with the TRS-80 microcomputer. Behavior Research Methods \& Instrumentation, 13, 674-676.

Grice, G. R., Canham, L., \& Boroughs, J. M. (1984). Combination rule for redundant information in reaction time tasks with divided attention. Perception \& Psychophysics, 35, 451-463.

Grice, G. R., Canham, L., \& Schafer, C. (1982a). Development of associative and perceptual interference. Perception \& Psychophysics, 32, 375-387.

Grice, G. R., Canham, L., \& Schafer, C. (1982b). Role of the response in associative interference. Bulletin of the Psychonomic Society, 20, 214-216.

Grice, G. R., Nullmeyer, R., \& SPIKer, V.A. (1977). Application of variable criterion theory to choice reaction time. Perception \& Psychophysics, 22, 431-449.
Grice, G. R., SPIKer, V.A., \& Nullmeyer, R. (1979). Variable criterion analysis of individual differences and stimulus similarity in choice reaction time. Perception \& Psychophysics, 25, 353-370.

Krueger, L. E., \& Shapiro, R. G. (1980). Repeating the target neither speeds nor slows its detection: Evidence for independent channels in letter processing. Perception \& Psychophysics, 28, 68-76.

McGill, W. J., \& GibBon, J. (1965). The general gamma distribution and reaction times. Journal of Mathematical Psychology, 2, 1-18.

TAYLOR, D. A. (1977). Time course of context effects. Journal of Experimental Psychology: General, 106, 404-426.

YEH, Y.-Y., \& ERIKSEN, C. W. (1984). Name codes and features in the discrimination of letter forms. Perception \& Psychophysics, 36, 225-233.

\section{NOTES}

1. Grice et al. (1982a) presented, in an appendix, a summary of the scaling procedures used with variable criterion theory. They also presented a summary of the race model, applicable to CRT, that deals with the relation between correct responses and errors. The earliest application of variable criterion theory to latency data was to distributions of conditioned responses by Grice (1972b). The earliest application to RT data was by Grice (1972a). The most complete account of the scaling procedures was by Grice, Nullmeyer, and Spiker (1977, pp. 432-434). The race model, a corrected form of an earlier version, was derived by Grice et al. (1979, pp. 354-357).

2. A response evocation characteristic (REC) is the relation between the scale values of two conditions plotted at corresponding times following stimulus onset. If this relation is linear, it implies that the two growth functions are of the same form. The slope of the linear regression line estimates the ratio of the standard deviations of the two criterion distributions. The intercept estimates the difference between the means of the criterion distribution. This relation permits the transformation of either set of scale values to the scale of the other. The REC is more fully described in the citations given in Note 1 . 